\title{
Germanica
}

\section{Der junge deutsche Film seit 1998. Innovative Impulse, Themen, Literaturadaption}

Le Jeune Film Allemand depuis 1998. Innovation, transformation de la littérature

\section{Volker Wehdeking}

\section{(2)enEdition}

\section{Journals}

Édition électronique

URL : http://journals.openedition.org/germanica/616

DOI : $10.4000 /$ germanica.616

ISSN : 2107-0784

\section{Éditeur}

Université de Lille

\section{Édition imprimée}

Date de publication : 1 juin 2009

Pagination : 107-122

ISBN : 978-2-913857-23-0

ISSN : 0984-2632

Référence électronique

Volker Wehdeking, «Der junge deutsche Film seit 1998. Innovative Impulse, Themen,

Literaturadaption », Germanica [Online], 44 | 2009, Online erschienen am: 01 Juni 2011, abgerufen am

10 Dezember 2020. URL : http://journals.openedition.org/germanica/616 ; DOI : https://doi.org/

10.4000/germanica.616

Ce document a été généré automatiquement le 10 décembre 2020.

(C) Tous droits réservés 


\title{
Der junge deutsche Film seit 1998. Innovative Impulse, Themen, Literaturadaption
}

\author{
Le Jeune Film Allemand depuis 1998. Innovation, transformation de la \\ littérature
}

Volker Wehdeking

Das hier gewählte Jahr 1998 für den Beginn einer neuen Phase im deutschen Film geht auf Tom Tykwers herausragend innovativen Film «Lola rennt» zurück, wo Elemente des Comic, Berlin-Thriller und Liebesmelodram eine tragikomische, postmoderne Genre-Mischung eingehen, eindringlich strukturiert durch eine dreifach wiederholte Handlungsschleife, einen loop. Die schauspielerische Leistung der Hauptdarsteller, Franka Potente und Moritz Bleibtreu kommen hinzu. Der dramatische Rettungslauf der Geliebten, beider zweifacher Liebestod mit vorausgehendem (und farbverfremdet magisch herausgehobenem) Gespräch über die Liebe und die letzten Dinge nach dem Liebesakt (Kamera-Aufsicht mit zoom) und einem als magisch unwirkliche Lösung inszenierten, ironischen Happy End sorgten für Tykwers Durchbruch, internationalen Publikumserfolg und unter anderen Auszeichnungen den Deutschen Filmpreis. ${ }^{1}$

Das Zusammenspiel von geschichtsträchtiger Kulisse und einer Präferenz für den zwischen Comicanleihen und Generationenkonflikt im psychologischen Realismus kontrastreichen, postmodernen Blick auf den Alltag eines jungen Liebespaars, der plötzlich aufgrund der Mafia-Kontakte des Helden in tödliche Bedrohung umschlägt, demonstriert diesen neuen Trend, dem bald das Etikett eines ,Jungen deutschen Films' als Erfolgsgeschichte anhaftete:

Lola rennt durch Berlin, von Kreuzberg/ Friedrichshain aus um den Nollendorfplatz, dann hetzt sie am Gendarmenmarkt vorbei und landet schließlich in der Friedrichstraße. Die Topographie dieses dreimal wiederholten Parcours ruft Filmgeschichte auf und Historie, aber der Film belässt es bei der Anspielung, bei dem Wissen um die Möglichkeit - und Unmöglichkeit - dessen, was da geschehen muss: das Leben ist ein grausames Spiel um Liebe und Geld (nicht umsonst ist rot die dominante Symbolfarbe dieses Films), und wir sind die Spieler ebenso, wie die, 
die da verspielt werden. Lola rennt ist ein Abgesang auf die im Berlin der 20er Jahre begonnene, verspielte Geschichte der Menschen dieser Stadt, es ist die Darstellung der Beliebigkeit ihrer Begriffe von Geld und Macht und es ist noch einmal ein Loblied auf die Kraft der Liebe. ${ }^{2}$

Die Zielgruppe dieses dritten wichtigen Tykwer-Films (nach «Die tödliche Maria» und «Winterschläfer») war die ,Generation Golf' bzw. ,Generation Berlin' der etwa zwischen 1965 und 1975 Geborenen, der Tykwer (geb. 1965 in Wuppertal) selbst angehört, und die Großstadtsymphonie war inspiriert vom MTV-Format. Das Gespür des jungen Regisseurs für Bewegung und Raum und die vielschichtige Erzählstruktur wurden ebenso beim Kritikerlob vermerkt, wie Tykwers in bisher allen Filmen (bis auf den in Hollywood-Dimension in Berd Eichingers Produktion 2006 illustrierend verfilmten Roman «Das Parfum») spürbare Reflexionen über «Zeitlichkeit und Kausalität» und neben dem Markenzeichen des «Herzpochens» die Demonstration der Macht des Zufalls und der Glaube an die «heilende Kraft» der Liebe. ${ }^{3}$

Die Dramaturgie des Drehbuchs und die Tempozunahme im Handlungsverlauf und den immer kürzeren Einstellungen folgen der Struktur einer Spirale. Die Symbolik der Spirale (als Bildmetapher mehrfach eingesetzt, etwa im Treppenhaus Lolas, gespiegelt in der Hauptfigur als Comic-Heldin auf nämlicher Treppe) visualisiert das ratlose «Umsich-selbst-drehen» der im Wettlauf mit der Zeit und in der Begegnung mit verhärteten Mitmenschen ihr Leben für den Freund Manni einsetzenden Liebenden.

Bevor ich mich den weiteren Filmen aus dem im Folgenden benannten, breiten Spektrum solcher Berlin-Themen (Tykwer filmt in beiden, ehemals durch die Mauer getrennten Teilen Berlins) und den bundesdeutschen Alltag nach dem Millennium darstellenden Kunstfilmen zuwende, möchte ich noch den auch mediennah und filmisch erzählten Trend in fiktionaler Gegenwartsprosa skizzieren, wobei auffällt, wie viele der hier aufgeführten, neuen Filme Literaturadaptionen sind: An möglichen, preisgekrönten und ambitionierten Filmen und ihren Regisseur(inn)en für meine Darstellung wären in Frage gekommen:

Caroline Link: «Jenseits der Stille» (1996), «Nirgendwo in Afrika» (2002), Sönke Wortmann: «Campus» 1998, Wolfgang Becker: «Das Leben ist eine Baustelle» (1997, Drehbuch mit Tom Tykwer), «Good Bye, Lenin!» (2003), Peter Kahane: «Bis zum Horizont und weiter» (2000), Andreas Dresen: «Nachtgestalten» (1999), «Halbe Treppe» (2002), «Sommer vorm Balkon» (2005), «Willenbrock» (2005), Hannes Stöhr: «Berlin is in Germany» (2001), Leander Haußmann: «Sonnenallee» (1999) und «Herr Lehmann» (2003), Hans-Christian Schmid: «Crazy» (2000), Gregor Schnitzler: «Solo Album» (2003), Fatih Akin mit dem transkulturellen, türkisch-deutschen Thema «Gegen die Wand» (2004) sowie Florian Henckel von Donnersmarcks «Das Leben der anderen» (2006). Die hier in Filme transformierten Texte stammen von den Autor(inn)en: Stefanie Zweig (Nirgendwo in Afrika, 2000), Dietrich Schwanitz (Der Campus, 1995), Christoph Hein (Willenbrock, 2000), Benjamin Lebert (Crazy, 1999), Benjamin v. Stuckrad-Barre (Solo-Album, 1998), Sven Regener und Thomas Brussig.

In den intertextuellen Anspielungen auf Autoren wie Irving, Carver, Welch und Ellis und auf Filme von Altman, Lynch und Tarantino zeichnet sich die ohnehin gemeinsame, längst erfolgte Überwindung der Mauer im Kopf bei den Jüngeren ab. Der veränderte mentale und mediale Blick lässt, ungeachtet der weiter bestehenden Unterschiede in der mémoire collective, die veränderte deutsche Wirklichkeit und ihre Mediensimulation in einem gemeinsamen literarischen Feld so beschreiben, dass die Foucault'schen ,Heterotopien' am Schwinden sind, auch wenn mancher Nostalgiker 
dies bedauern mag. Eine Normalisierung mit dem entspannten Verständnis eigener regionaler Identität und Thematik ist die Folge (etwa in Ingo Schulzes neuen Erzähltexten «Handy» und dem Roman «Adam und Evelyn», 2008, mit ostalgischer Sicht).

7 In drei Romanen nach dem Millenium wird dieser Umschlag in die Dominanz ,privater', aber nicht unpolitischer Alltagsperspektive auch im Stadtpanorama Berlins besonders deutlich: in Ulrike Draesners neorealistischem Liebes- und Familienroman «Mitgift» (2002) um die Kunsthistorikerin Aloe, ihre hermaphroditische Schwester und den Astronomen Lukas zwischen Oxford, München und Berlin. Der Berlin-Besuch der Protagonistin Ende der 90er Jahre zeigt den Potsdamer Platz im Umbau. Die «Stadtbewohner» nennen ihn nur «das Loch». Der Besuch der Metropole mündet jedoch eher in Liebeskummer und den Diskobesuch, um den nach Chile ans Observatorium gegangenen Freund $\mathrm{zu}$ vergessen, das Stadtpanorama erscheint wie durch ein umgekehrtes Fernglas, wird dem Privaten nachgeordnet und den Medien:

Aloe lief und fuhr, kreuz und quer, durch die Stadt. Alles war kleiner als im Fernsehen. Von der S-Bahn aus sah die eingerüstete Kuppel des Reichstages aus wie ein überdimensioniertes Strickzeug. Die sogenannten Quartiere in der Friedrichstraße klebten am Gehsteig, als wären sie koupierte Legohäuschen. [...] Überall standen Liebespaare, an jedem Bahnsteig nahmen sie Abschied [...] Küsse, Küsse. [... Der Potsdamer Platz, d. V.] in der Dämmerung, von riesigen Scheinwerfern in kegelige Schnitte zerlegt. Entworfen für eine Zukunft, die Verkehrsstrom war. Grünbraunes, trübes Wasser, in dem Betonfundamente schwammen, darüber balancierten Kräne mit weit ausgestreckten Schnäbeln. Sony hatte angefangen, die Gruben für seine Türme und Rotunden, seine Filme und seine Realität auszuheben, die Mulden der Tage und Nächte, in denen man in Zukunft essen, kaufen, ficken wollte. Aloe stand über einer Behelfsbrücke aus Eisen und schaute Tauchern zu, die in dunklen Neoprenanzügen in die schlammige Baubrühe hinabgelassen wurden, große Lampen am Kopf. Sie wollte sich von Lukas ablenken. ${ }^{4}$

Die Dominanz der neuen Mediensimulation könnte nicht deutlicher erscheinen, als in der Beobachtung, dass die Realität der markanten Berliner Gebäude und Plätze «kleiner» wirkt als die im deutschen Leitmedium Fernsehen gezeigten Bilder. Und nicht die architektonischen Symbole der Historie sind das Ziel, sondern Sinnenlust.

Im autothematischen Roman der Malerin Cornelia Schleime, «Weit fort» (2008) über ihre Enttäuschung in gescheiterten Bindungen wird dem Gefühlssturm nach dem Abschied auch die mögliche Stasi-Verstrickung des Partners untergeordnet. Es geht nur um die vermisste Aufrichtigkeit. So dominiert auch hier das private Berlinbild, auch wenn die Topographie einer Jugend vor dem Mauerfall in «Hessenwinkel, dem Nobelviertel von Ostberlin» und eine «Villa am Dämmeritzsee» als romantisch erinnertes «Klein-Venedig» eine Rolle spielen. Der dubiose Freund, wohl ähnlich verstrickt wie der Jugendfreund Sascha Anderson (im Roman nicht genannt, aber nahe der authentischen Beziehung) nach Aufdeckung seiner Stasi-Bespitzelung, als ehemaligen Ostberliner, der jetzt TV-Journalist in Regensburg ist, verlässt sie ohne Abschied, ein indirektes Schuldgeständnis. Beziehungsgeschichten haben die Ereignisgeschichte abgelöst.

Mit dem vierten Roman nach seinem Debüt 1987, «Teil der Lösung», 2007, hat der 1956 in Krefeld geborene, in Berlin lebende Ulrich Peltzer ein neues Kapitel im Stadtpanorama Potsdamer Platz, Kreuzberg, Heinrichplatz und Paris-Belleville für die postideologische Gesellschaft aufgeschlagen. Es ist ein fulminanter, zeitkritischer Berlin- und Liebesroman, der auch medienrelevant die elektronischen 
Überwachungsmedien beschreibt und Filmästhetik ins Erzählverfahren in kurzen harten Schnitten einbezieht. In seinem polyphonen Stadtpanorama geht es nun aber, nach der Erzählung Bryant Park, 2002 (als Peltzer in New York lebte und den 11. Sept. 2001 als ,Riss durch die Welt' erlebte), um Themen des Überwachungsstaats, der Globalisierung, des Terrorismus von 1968 und heute (die Brigade Rosse heute im Pariser Exil), um Engagement und Liebe.

Der Generationenwechsel dokumentiert sich bei von Donnersmarcks mit vielen Preisen, zuletzt dem Oscar (Best Foreign Film 20075) bedachten Film auch im Vergleich zu Volker Schlöndorff (geb. 1939) und dessen lange zuvor mit dieser Auszeichnung hervorgehobenen Blechtrommel-Verfilmung von 1979 in der Ära des New German Cinema mit Fassbinder, Wenders, Kluge, von Trotta und Herzog. Der neuerlich vielbeachtete Junge deutsche Film seit Tom Tykwers Lola rennt, 1998 geht im Medienwechsel mit der deutschen Gegenwartsliteratur eine für beide Seiten produktive Symbiose ein (etwa der Filme nach Regener, v. Stuckrad-Barre und Brussig im MTV-Format), vor allem in Tykwers Adaption von Süskinds Das Parfum 2006, aber auch Andreas Dresens Sommer vorm Balkon oder Caroline Links Nirgendwo in Afrika und Hans-Christian Schmids CrazyAdaption Benjamin Leberts.

Der deutsche Gegenwartsfilm findet ungeachtet einer Rückkehr zum Unterhaltungsfilm nach dem Fall der Mauer in den frühen 90er Jahren, als man die harmonisierenden Effekte des Genre-Kinos im Gefolge von Hollywood wieder entdeckte und einige Beziehungs- und Yuppie-Komödien nach der Bestseller-Autorin Hera Lind drehte (Peter Timm, Sönke Wortmann), wieder zu politischen Themen in der oral history privater Alltagsdimension zurück. Tykwer und andere signalisieren, parallel zum derzeitigen fiktionalen Vorherrschen der Thematik des Deutschen Herbst und der 68er Terroristen in der Literatur (Bernhard Schlink: «Das Wochenende» 2008, Ulrike Draesner: «Spiele» 2005, Inka Parei: «Was Dunkelheit war» 2005) in der Literatur durch das Filmprojekt «Deutschland 09» zur Lage Deutschlands im Herbst 2008 in einer Film-EpisodenZusammenarbeit wie in «Deutschland im Herbst» (1978), diesmal von Tykwer, Akin, Becker, Graf, Karmakar, Sylke Enders, Nicolette Krebitz, Isabelle Stever und weiteren Regisseuren den Brückenschlag zum New German Cinema. Auch Schlöndorff und von Trotta haben in den Babelsberger Studios mit Filmen zur Kriegs- und HolocaustThematik («Der neunte Tag», "Rosenstraße», sowie jüngere Regie-Arbeiten von Vilsmaier und Hirschbiegel) die zeitkritische Sicht des New German Cinema fortgesetzt, nunmehr in ,privater' Perspektive. Aus den oben aufgeführten, wichtigen Filmen des Jungen deutschen Films wähle ich, um nicht ins bloße Summieren zu geraten, nach Tykwers Initial-Film zur näheren Analyse zwei aus, die zum Post-DDR-Thema eine westliche und östliche Perspektive einbringen.

\section{Florian Henckel von Donnersmarcks «Das Leben der anderen» (2006). Genre-Mischung um Kern-Themen der Literatur. Lauschangriff und Widerstand in der späten DDR. ${ }^{6}$}

Ein auf den ersten Blick dem Zerbrechen und Standhalten von Menschen unter dem Druck der Stasi zur DDR-Zeit der Erstarrung gegenüber glasnost und perestroika gewidmeter Polit-Thriller erweist sich auf den zweiten Blick als ein besonders nah an 
den Anliegen der Literatur und Kunst heranführendes Psycho-Drama. Subtil wird aus der Psyche der Hauptfiguren in verschiedener Motivation der innere Normen-Konflikt unterdrückter Literatur und Kunst und deren inhärente Disposition zur Freiheit durch die skandalösen (weil hier nicht nur ideologisch, sondern auch aus Machtmissbrauch zugunsten der sexuellen Wünsche eines Ministers der Kulturabteilung des ZK motivierten) Menschenrechtsverletzungen der Stasi greifbar, die dabei über Leichen geht. Der Theater-Regisseur, der sich, weil in einem inoffiziellen ,Berufsverbot' seit Jahren kaltgestellt, das Leben nimmt, die Schauspielerin, die unter Druck gesetzt, vermeintlich einen Schriftsteller im Widerstand, ihren geliebten Lebenspartner, verrät und, in zunehmendem Verlust ihrer Selbstachtung nach den Pressionen des Ministers verzweifelt vor ein Auto läuft, sind die Opfer. Von daher wird deutlich, dass dieser Film von Donnersmarcks auch ein Kontext-Film von Literatur, nämlich eminent literarischer Themen ist: gelten doch als ,innerliterarisch' besonders die Beziehung von Leben und Werk als die ,bruchlose Existenz' zwischen intellektueller Disposition und Handeln, eine Kernvorstellung des Existentialismus. Es sind vor allem hier die Fragen der Gesinnungsfreiheit und Selbstverwirklichung von Kunst und Literatur unter Regisseuren, Schauspielern und Schriftstellern. Die nähere Analyse des Filmdramas ergibt, wie dieses spezielle, und internationale bildungsbürgerliche Publikum die besonderen Themen der Literatur und ihrer Unterdrückung innerhalb der Lebenswelt der späten DDR (im Rückblick von fast zwei Jahrzehnten) wahrnehmen konnte, wie die handfeste Spannungsregie und die Einbettung in das Genre des Thrillers noch eine viel breitere Zielgruppe $\mathrm{zu}$ erreichen vermochte. Die als Lesedrama mit den Filmillustrationen attraktive Buchveröffentlichung zeigt bei näherer Lektüre der Filmeinstellungen auch, wie sich neben den Drehbuchqualitäten einer grundsätzlichen Dreiteilung in Exposition, Konfrontation und Auflösung des Konflikts zwischen den drei Hauptfiguren auch die Qualität eines tektonisch gebauten Dramas herauskristallisiert. Dessen aristotelische Einheiten von Zeit, Ort und Handlung sind ebenso ablesbar, wie die opulenten Regieanweisungen zu den Filmeinstellungen nur in diesem Medium als Film-Drehbuch typisch sind: die eingestreuten Filmbilder im Drehbuch (als Buchausgabe und Film-Tie-In bei einem intellektuell so renommierten Verlag wie Suhrkamp) und die ausschließlich im Film darstellbaren, weil Fotorealismus mit maximaler szenischer Beweglichkeit in den Doppelperspektiven bei den StasiÜberwachungszenen und den Straßenszenen in Ost-Berlin umsetzenden Einstellungen.

Der 1973 geborene von Donnersmarck lernte nach einem Regie-Praktikum bei Richard Attenborough an der Münchner Hochschule für Film und Fernsehen Drehbuch und Filmregie. Die Idee und das Drehbuch zu Das Leben der anderen beschäftigten den Regisseur mehr als fünf Jahre bis zur Realisierung des Films, vom aufwendigen Einwerben von Mitteln für den Erstling als Langspielfilm ganz abgesehen. Unter dem Kreativitätsdruck der Filmhochschule, bereits im ersten Semester vierzehn Filmentwürfe abliefern zu sollen, kam ihm die wie ein Leitmotiv wirkende (im Film dann als Geschenk des befreundeten Regisseurs erhaltene) Musikpartitur „Die Sonate vom Guten Menschen“ als auslösende Grundidee und magisches ,Urbild' beim entspannten Anhören der Mondschein-Sonate, gespielt von Emil Gilels:

Da plötzlich kam mir etwas in den Sinn, was ich einmal bei Gorki gelesen hatte, dass nämlich Lenin über die „Appassionata“ gesagt habe, dass er sie nicht oft hören könne, weil er sonst „liebevolle Dummheiten sagen und den Menschen die Köpfe streicheln“ wolle, auf die er doch „einschlagen, mitleidlos einschlagen“ müsse, um seine Revolution zu Ende zu bringen. Mit der „Appassionata“ war es mir nie so gegangen, aber bei der „Mondschein-Sonate“ konnte ich Lenins Aussage auf einmal 
verstehen: Manche Musik zwingt einfach dazu, das Menschliche über die Ideologie zu stellen, das Gefühl über die Prinzipien, die Liebe über die Strenge. Ich fragte mich, was wohl geschehen wäre, wenn man Lenin hätte zwingen können [...], die „Appassionata“ für die revolutionäre Sache hören zu müssen. Während ich darüber nachdachte, drängte sich mir ein Bild auf: die Halbnahe eines Mannes in einem trostlosen Raum; er hat Kopfhörer auf den Ohren, durch die eine wunderbare Musik klingt. Und als dieses Bild da war, stürzten die Gedanken auf mich ein: der Mann hört diese Musik nicht zum eigenen Vergnügen, sondern weil er jemanden belauschen muss, einen Feind seiner Ideen, aber einen Freund dieser Musik. Wer ist dieser Mann, der da sitzt? Wen belauscht er? Die Fragen kamen im Rausch, ebenso die Antworten, und innerhalb weniger Minuten stand das gesamte Grundgerüst zu „Das Leben der anderen“. [...] Keine acht Jahre später war er fertig“."7

Schon die Entwicklung dieses magischen Bildes, im Gedankengang politisch-ethisch (Lenin) und in ästhetischer Kontrafaktur der Machtfrage erweist die Bedeutung von Donnersmarcks Bildungsstationen: etwa der Aufenthalt in St. Petersburg, und für die Dialektik mit der Disposition zur existentiellen Entscheidung der längere Aufenthalt während der Arbeit am Drehbuch bei einem Onkel im Stift Heiligenkreuz bei Wien, dem dortigen Zisterzienser-Abt Gregor Henckel-Donnersmarck. Für eine weitere Kernszene, «die große Jerska-Szene» gibt es den Beleg der Proben mit Sebastian Koch in der Rolle des Autors und Protagonisten Georg Dreyman, den Koch als Menschenfreund und zugleich distanzierten, genauen Beobachter empfindet. Auf einer Party bei Dreyman erscheint der durch die lange Kaltstellung (wegen seiner mangelnden Linientreue) bereits verbitterte Regisseur Jerska, für den sich Dreyman anfangs vergebens beim Minister Hempf einsetzte. Er gibt Dreyman als Geschenk eine Klavierpartitur «mit dem seltsamen Titel» (Regieanweisung, LA, S. 57) «Die Sonate vom Guten Menschen».

Als man Dreyman, der ungeachtet der Verachtung Hempfs für Jerska diesem noch Hoffnung zu machen versucht, einige Tage später mitteilt, dass Jerska sich erhängt hat, spielt er die im Film vom französischen Komponisten Gabriel Yared genial in getragenenen Triolen mit melancholisch absteigendem Motiv gestaltete Sonate; er spielt sie «in ihrer vollen Schönheit und Melancholie». Die Lebensgefährtin ChristaMaria, die sich am Klavier hinter ihn stellt, «merkt seine Erschütterung» (LA, S. 76). Nun wird er den mit den Freunden besprochenen Widerstandstext für eine Westveröffentlichung schreiben; und der ihn abhörende Stasi-Hauptmann Wiesler (Ulrich Mühe) erlebt auch seine entscheidende Wendung zum Guten bei Dreymans Frage: «Kann jemand, der diese Musik gehört hat, wirklich gehört hat, noch ein schlechter Mensch sein?» In der Regieanweisung heißt es über den Close-Up «von vorne. Auf seinem Gesicht liegt ein noch nie gesehener Ausdruck» (LA, S. 77).

17 Wiesler, der $\mathrm{zu}$ Anfang des Drehbuchs in einer Gefängnisszene des StasiUntersuchungsgefängnisses Hohenschönhausen im November 1984 einen Häftling so lange verhört, bis dieser nach vierzig Stunden am Ende seiner Kraft und Nerven alles gesteht, was man von ihm hören will, und diesen Mitschnitt ideologisch verbohrt den Schülern der Stasi-Hochschule Potsdam-Eiche als beispielhaftes Verhalten präsentiert, erlebt in der Mitte des Dramas, in der inneren Handlung, seine Umkehr zum unauffälligen Freund der Liebenden, des Schriftstellers und der vom Minister begehrten Schauspielerin. Er wird ihm nun Linientreue attestieren und Christa-Maria Sieland weitere Treffen mit dem unsäglichen Minister ausreden. Dass sich Wiesler, bei seiner Abhörroutine Brecht lesend, die berühmte Liebesballade «Erinnerung an die Maria A.» mit Dreymans Stimme vorstellt, bestätigt die innere Nähe beider und Wieslers Wandel (LA, S. 75). 

eine Premieren-Party und neben den glücklichen und erfolgreichen Liebenden beim Tanz die sich zuziehende Schlinge der Gesinnungsschnüffler auf drei Ebenen der StasiHierarchie. Weitere anwesende Journalisten (Hauser, überwacht durch den IM und Regisseur Schwalber) werden verdächtigt. Der lüsterne Minister sucht Material gegen den Lebenspartner der Sieland. Die Dimensionen der Anti-Utopie eines Orwell-Staates werden in den Einzelaktionen des Lauschangriffs in einer technisch perfekten Überwachungszentrale auf dem Dachboden über der Schriftstellerwohnung deutlich. Die Langzeitwirkung des faktischen ,Berufsverbots' auf den einstmals berühmten Theater-Regisseur Albert Jerska, der nach sieben Jahren dieser Behandlung durch die Kulturabteilung des ZK sich das Leben nimmt, stimmt zu diesem tödlichen Überwachungsbild.

19 Auf der Party zu Georg Dreymans 40. Geburtstag wirft Hauser dem Autor allzu devote Anpassung vor. An einem der kommenden Abende fällt Wiesler, der Dreyman unter dem Codewort «Lazlo» abhört, das Aussteigen der Schauspielerin aus dem Ministerwagen, einer großen schwarzen Wolga-Limousine auf. Die zuvor gezeigte Vergewaltigung, bei der die attraktive Sieland in den Wagen steigt «wie auf ein Schafott» (LA, S. 64) und der schwerleibige Fünfzigjährige die mit Rücksicht auf ihre Theaterkarriere und Liebe zu Dreyman nur undeutlich Widerstrebende halb entkleidet, dann «wie ein Tier» an sich presst, und sich an ihr vergeht (nachdem er ihr wiederholt mit der Limousine auflauerte), lädt die Konfrontation zwischen Tätern und Opfern im nächtlichen Ost-Berlin mit Thriller-Dimensionen der Spannung auf.

Wiesler durchschaut die hässliche Intrige, erfährt aber neuen Druck durch den Vorgesetzten, verdächtiges Material bei Dreyman zu finden. Dennoch spricht er bei einem Zufallstreff mit der Sieland in einer nächtlichen Bar ihr auf eine Weise Mut zu, die die Bitten des liebenden Schriftstellers, den Minister, von dem er weiß, zu ignorieren, verstärkt. Die Tablettenabhängigkeit ist ein erschwerendes Moment für die Schauspielerin, aber sie zeigt nun Ich-Stärke und versetzt den mächtigen Lüstling. Nach Jerskas Freitod beschließt Dreyman, einen Artikel über die Selbstmordrate in der DDR über Hauser in den Westen, dem Spiegel, zuzuleiten und es kommt zu heimlichen Treffen mit dem Effekt, dass ein Spiegel-Redakteur eine Mini-Schreibmaschine einschmuggelt um Dreymans Schriftbild zu tarnen. Da Christa-Maria Sieland den Minister nicht mehr trifft, sucht dieser in seinem Zorn einen Weg, über ihre Tablettensucht und ein Stasi-Verhör erst durch den Vorgesetzten, dann durch Wiesler selbst das Versteck der Schreibmaschine des inzwischen veröffentlichten West-Artikels herauszufinden und den Liebesverrat zu erzwingen. Dies gelingt auch teilweise, da man ihr das Aus jedweder Theaterlaufbahn androht und sie sich als IM verpflichten muss, sowie Dreyman als Autor des Spiegel-Artikels verrät. Sie darf aber auf das Geheimversteck hoffen, so dass keine Beweise Dreyman gefährlich würden. Beim nächsten Verhör am Morgen darauf erkennt Christa-Maria in Wiesler ihren wohlwollenden Ratgeber des nächtlichen Treffens und verrät ihm daher alles, auf seine Mithilfe hoffend; dieser kann zwar noch rasch die Maschine aus dem Versteck entfernen, aber die wiederholten Stasi-Besuche haben das Vertrauen Dreymans angeschlagen und die verzweifelte Schauspielerin rennt panisch aus der Wohnung vor einen LKW. Sie stirbt in Dreymans Armen, ohne dass ein Beweismittel gefunden wurde. Dies kann Wiesler der Sterbenden noch zuflüstern. Der tragische Freitod der Geliebten als direkte Folge der Stasi-Machenschaften wird in einem emotionalen Höhepunkt des 
Films, in der Tonunterlage von einer elegischen, klassisch-modernen Orchesterkomposition atmosphärisch dicht gestützt, zum endgültigen Wendepunkt für Wiesler und beendet die Verfolgung Dreymans. Der Verdacht der Vorgesetzten fällt auf Wiesler, der auf einen niedrigen Posten zur Briefüberwachung versetzt wird, bis die Mauer fällt. Obwohl ihm der Vorgesetzte zwanzig Jahre subalterne Qual bis zur Pensionierung voraussagt, schaltet eine Einblendung den Zeitsprung bis zum Mauerfall: «Vier Jahre und sieben Monate später». Wiesler kann die Fron des stündlichen Aufdampfens von 600 Briefen in einem öden Stasi-Büro verlassen.

21 Nach der tragisch endenden Konfrontation von Tätern und Opfern und den Szenen der großen Emotionen gibt es die Auflösung mit dem weiteren Schicksal Dreymans, der zwei Jahre nach dem Mauerfall bei einer Neuinszenierung seines Eröffnungs-Stückes «Gesichter der Liebe» den Saal im nicht überwundenen Schmerz verlässt. Er trifft zu seiner großen Betretenheit auf den ehemaligen Minister Hempf, der ihn ambivalent auf Christas Freitod anspricht, und ebenfalls Verlust-Gefühle heuchelt. Aber obwohl Hempf noch eine Stelle hat und den eigenen Sohn als Abgeordneten der PDS lancieren konnte, kann er nicht auf Dreymans Verständnis rechnen, als er resigniert zu der ostalgischen Einsicht findet: «Was soll man auch schreiben in dieser BRD: nichts mehr da, woran man glauben kann, nichts mehr, wogegen man rebellieren kann. [...] Es war schön in unserer kleinen Republik. Das verstehen viele erst jetzt» (LA, S. 149). Der Zynismus des Ministers wird kaum gemildert durch die Auskunft (auf Dreymans Frage nach seiner Überwachung), dass man ihn umfassend und dauernd abhörte; aber er weiß nun von seinem Gönner unter den Stasi-Leuten.

Das versöhnliche Nachspiel erweist dann das eigentliche Thema nochmals auf der ethischen Seite der Politik und Liebe, und in den psychologischen Nuancen integren Handelns im Orwell-Staat. Wo Christa-Maria Sieland den Liebesverrat als Urteil in den Augen des liebenden Schriftstellers nicht ertragen hätte, nachdem sie ihn doch zum Widerstand ermutigte, und sich im Affekt das Leben nimmt, kann Dreyman nun seinem Retter Wiesler auf seine Weise danken: mit einem Roman über die gesamten, tragischen Erlebnisse der Liebenden, und dem Dank an seinen «Schutzengel» unter all den OrwellMittätern mit der Dienst-Bezeichnung HGW XX/7, die er aus den Gauck-Akten erfuhr, ebenso wie die heroische Haltung seiner Geliebten. Ironischerweise war, wie er in den Akten liest, der Abhörende $\mathrm{zu}$ seinem Schutz so weit gegangen, statt der Widerstandstexte ein Drama zum 40. Jahrestag der DDR über den heroischen Lenin zu fingieren. Donnersmarcks Ausgangsbild wird also auch hier eingeholt. Dreymans Roman liegt, wiederum zwei Jahre später, in allen Berliner Buchhandlungen, als «der erste Roman des großen Dramatikers» mit dem Plakat des Autors und dem Titel: «Die Sonate vom Guten Menschen». Wiesler erkennt ihn auf dem Plakat, betritt die Buchhandlung und findet die Widmung an dessen Dienst-Nummer, «in Dankbarkeit» (LA, S. 158). «Erschüttert» liest Wiesler, der den Tod der bewunderten Schauspielerin ebenfalls nicht verwunden hat und auf seine Karriere verzichtete, die Widmung. Als der Buchhändler den Roman als Geschenk verpacken will, sagt er «Nein ... es ist für mich». Auch wenn Donnersmarcks vielschichtige Botschaft über die Kraft des Guten und der Kunst inmitten ihrer Unterdrückung und die Vermeidung einer Schwarz-WeißZeichnung von Tätern und Opfern hier versöhnlich endet, bleibt doch der Grundton der Melancholie über den Verlust der großen Liebe aufgrund der staatlichen Pressionen des Systems. Seinen Retter unter den Stasi-Männern anzusprechen, brachte Dreyman bereits zwei Jahre zuvor nicht über sich. Als er ihn zufällig auf der Karl-Marx-Allee 
während einer Taxi-Fahrt erblickt, steigt er zwar aus, überlegt es sich aber anders: der Grundton bleibt in dieser Distanz und Trauer.

Die postmoderne Metafiktion der Entstehung eines Widerstands-Berichts über die DDRSelbstmorde und die Entstehung eines Romans im Filmdrama, mit dem Titel «Die Sonate vom Guten Menschen» im gelungenen Genre-Mix des Drehbuchs zeigt die Kontext-Qualität dieses Films zu Anliegen der Literatur. Es geht um die nicht manipulierte, bei allen Heterotopien soziopolitischen Umfelds behauptete Gesinnungsfreiheit ohne Pressionen und Zensur. Das Trauma der DDR-Bürger kommt zur Wirkung, die unter Macht und Ohnmacht der repressiven Partei-Diktatur im Orwell-Staat zu leiden hatten. Das macht bereits die Wahl der erzählten Zeit mit dem Datum 1984 in seiner Anspielung auf den Orwell-Titel (der wiederum die dystopischen Gefühle der Nachkriegszeit im umgedrehten Datum von 1948 meinte). Im Raum von Albert Jerska, der an seinem siebenjährigen Kaltgestelltwerden ohne Wirkungsmöglichkeiten als einst berühmter Regisseur zerbricht, wird das bildungsbürgerliche Potential der Aufklärung und des freien Wortes anhand Donnersmarcks Beschreibung seiner Wohnung in der Regieanweisung deutlich: ein großer Raum, «der auf beiden Längsseiten, die von Tür zum Fenster führen, bis zur Decke vollgestellt ist mit Büchern. Der Raum hat etwas von Fausts Studierstube» (LA, S. 45). Kunst und Literatur sind die Gegengewichte zur Ideologie der Unterdrückung.

\section{Thomas Brussig: «Sonnenallee» (1999) - vom Drehbuch zur Erzählprosa}

Die neue intermediale Nähe von Erzählprosa und Filmdrehbuch ist in der Zusammenarbeit vom Regisseur Leander Haußmann (geb. 1959) mit dem Autor Thomas Brussig (geb. 1965) am Film «Sonnenallee» (1999) und dem erst danach geschriebenen Roman besonders sinnfällig. Brussig galt seit «Helden wie wir» (1995), einem satirischen Entwicklungsroman über den naiven Außenseiter-Helden (mit Eltern bei der Stasi) zur Vorgeschichte des Mauerfalls, als der ,ostmoderne' Neorealist. Bereits 1996 hatte Brussig die Idee, aus einer Schul- und Liebesgeschichte der 70er Jahre in der DDR, im Schatten der Mauer, einen Film zu machen, perfekt und allegorisch lokalisiert: «Am kürzeren Ende der Sonnenallee» bezog sich auf eine Berliner Straße, die sich über vier Kilometer und zum Großteil im Westen Berlins erstreckte und durch die Mauer so geteilt war, dass Westtouristen von einer Aussichtsplattform den Ostalltag voyeuristisch mitverfolgen konnten. Er rief daher Haußmann an, und beide verabredeten die Zusammenarbeit am Drehbuch über die erste Mauerkomödie. ${ }^{8}$ Das ursprüngliche Zielmedium war hier also der Film, und der ,Übersetzungsvorgang', von dem heute in der Forschung bei Filmadaptionen von Texten ausgegangen wird, ${ }^{9}$ verlief vom Film zum Buch. Die authentischere, wohl auch gelungenere Fassung, geben das Drehbuch und der danach gedrehte Film. ${ }^{10}$

Es entstand eine postmoderne Nummernrevue, zu der in Babelsberg auf dem Filmgelände eine als Kulisse erkennbare Pappmauer aufgebaut wurde, hinter der nun die Thematik von Schulalltag, Tanzstunde, erster Liebe, Sehnsucht nach Levi`s Jeans, Springsteen und den Rolling Stones, Coca-Cola und der allmählich zur Herbstrevolution im Osten führenden Mentalität jugendlicher Emanzipation vom kasernierten Lebensgefühl anhand kleiner Widerstandsakte der DDR-Bevölkerung sinnfällig wurde; in den Worten des Autors war seine Intention: 
[...] die DDR als Kulisse zu benutzen. Die DDR hinterlässt nichts, außer der Mauer und der Tatsache, dass sich eine ganze Gesellschaft so gegen ihre eigenen Interessen organisiert hat. Keiner wollte die Mauer, trotzdem hat es sie immerhin achtundzwanzig Jahre gegeben. Der DDR ein Museum bauen? Eher dem eigenen Erwachsenwerden. Meine Kindheit hat in der DDR stattgefunden. Das macht die DDR nicht besser. Aber ich erinnere mich trotzdem gerne an die Kindheit. [...] Ich hatte das Gefühl, dass ,Sonnenallee' etwas im Publikum trifft, das dazu führt, dass jeder plötzlich anfängt, sich zu erinnern, egal, ob er im Osten oder im Westen groß geworden ist - er bekommt einfach Lust, etwas zu erzählen. Und das geht über den Film hinaus. " ${ }^{11}$

Die hier deutliche Zielgruppenbewusstheit des Autors wurde in der großen Resonanz des Films und Romans, gerade auch unter den Jüngeren mit Sinn für die Pluralität der Diskurse bestätigt. Die Geschichte um die Familie Kuppisch ist rasch erzählt. Höhepunkte der ,Nummernrevue' zeigen die Mutter, die einen gefundenen West-Paß vor dem ABV (Abschnittsbevollmächtigten) verheimlicht, um dann doch nicht damit über die Grenze am nahen Schlagbaum zu kommen, weil sie die Familie nicht verlassen kann. In der Schule entsteht über der Liebe zur schönen Miriam beim Sohn Michael ein mehrteiliges Tagebuch in Heften, mit denen er gegen die Attraktionen von Miriams anderem Verehrer, einem Westplayboy mit schickem Wagen (der aber nur Hotelportier und Parkwächter im «Schweizer Hof» ist) anzuwerben versucht, mit zunehmendem Erfolg. Michas Freund, der Stones-Fan Wuschel versucht, im Schwarzhandel ein Doppelalbum der Rolling Stones zu kaufen; als der ABV in einer Kurzschlussreaktion beim Ausfall der Straßenbeleuchtung (ironischerweise weil ein westliches Hi-Fi-Gerät ans ostdeutsche Stromnetz angeschlossen wurde) auf den vermeintlich Republikflüchtigen schießt (der eigentlich einen auf die Schienen geflatterten Liebesbrief suchen hilft), wird ihm dadurch das Leben gerettet, dass nur das Doppelalbum getroffen wurde, und er sich rasch von seiner Ohnmacht erholt. Der Westonkel Heinz schmuggelt immer die bereits in der DDR käuflichen Dinge in den Osten, bis er, der immer vor dem Asbest in DDR-Plattenbauten warnte, groteskerweise selbst an Lungenkrebs stirbt. Um ihm eine Familienfeier zu ermöglichen, schmuggelt Frau Kuppisch seine Asche in einer Kaffeedose in den Osten. Seine Nebenrolle bestand darin, die «Westverwandtschaft» (AS, S. 172) zu verkörpern, und damit eine einseitige West-Perspektive des Films zu konterkarieren. Die Party mit dem Freund Mario, der wegen wiederholter Unbotmäßigkeit aus der Schule fliegt und sich daher, als werdender Vater auf den Lebensunterhalt angewiesen, dem Stasi anschließen muss (was zu einer verzweifelten Prügelei mit dem enttäuschten Freund Micha führt), und seiner Existentialistin, läuft immer mehr aus dem Ruder, bis alle Komparsen in einer Art Ballett am Schlagbaum zu den Klängen von Rockmusik den Tanz in die Freiheit üben; der schwarzweiße Abspann zeigt die historische Weggerücktheit dieser Befreiungsanfänge am noch unüberwindlichen Schlagbaum an der Mauer, damals in den 70er Jahren.

Der nach dem Drehbuch zu Ende geschriebene Roman Am kürzeren Ende der Sonnenallee $(1999)^{12}$ enthält noch deutlichere historische Allegorien, um eine einseitige ostalgische Lektüre zu vermeiden und die Ironie-Signale grotesk zu verstärken. Gleich eingangs wird die Teilung der Sonnenallee «auf der Potsdamer Konferenz im Sommer 1945» (AS, S. 7) durch Stalin, Truman und Churchills Intervention beim Zigarrerauchen beschlossen; zugleich ist dies eine humorvolle Brussig-Illustration zu Hermann Lübbes Historiographie-These, dass Geschichte sich nicht systemrational, sondern nur erzählend mit allen Widersprüchen darstellen lässt. 
So muß es gewesen sein, dachte Michael Kuppisch. Wie sonst könnte eine so lange Straße so kurz vor dem Ende noch geteilt worden sein? Und manchmal dachte er auch: Wenn der blöde Churchill auf seine Zigarre aufgepasst hätte, würden wir heute im Westen leben. [...] Genauso wenig gewöhnte er sich an die tägliche Demütigung, die darin bestand, mit Hohngelächter vom Aussichtsturm auf der Westseite begrüßt zu werden, wenn er aus seinem Haus trat - ganze Schulklassen johlten, pfiffen und riefen ,Guckt mal, 'n echter Zoni!' oder ,Zoni, mach mal winke, winke, wir wolln dich knipsen!' (AS, S. 9).

Noch deutlicher auf eine eher zeitkritische und satirische als nostalgische Deutung des Ganzen beim Leser angelegt ist die Schlusssequenz des Romans, in der ein deutlich an seinem großen «Muttermal auf der Stirn» erkennbarer Gorbatschow «Wunder vollbringt», indem er Wettergott spielt, dann Geburtshelfer, schließlich auch noch den Trabi repariert. Der in Marios altem Trabi ihre Wehen erleidenden Elisabeth zeigt der «Wunderrusse» in wenigen Augenblicken ein «fertig gewickeltes Neugeborenes», bevor er lachend mit seinem Staatskonvoi entschwindet (AS, S. 156). An dieses natürlich auf Glasnost, Perestroika und DDR-Ende voraus weisende Politmärchen, das nur der Roman enthält, nicht Drehbuch und Film, schließt sich Brussigs Betrachtung über die trügerisch nostalgisch arbeitende, also verdrängende «Erinnerung» an: «Wer wirklich bewahren will, was geschehen ist, der darf sich nicht den Erinnerungen hingeben» (ebd.), ist ein die einseitig ostalgisch tendierende Rezeption im Ost-Feuilleton konterkarierendes Caveat, das aber durch die versöhnlichen Folgesätze über den Wunsch, «einen Frieden mit der Vergangenheit zu schließen», nicht als die intendierte Unterscheidung zwischen kollektiver, korrekter mémoire und privater, subjektiver Erinnerung des DDR-Alltags gelesen wird: «in dem sich jeder Groll verflüchtigt und der weiche Schleier der Nostalgie über alles legt, was mal scharf und schneidend empfunden wurde. Glückliche Menschen haben ein schlechtes Gedächtnis und reiche Erinnerungen» (AS, S. 157). Der Film kann diese durch Mehrfachcodierung intendierte, die Befreiung hinter der Mauer ersehnende (und durch die ,Existentialistin' deutlich an Sartres größtmögliche Freiheit aller in der Selbstwahl festgemachte) Mentalität der Jugendlichen, die dennoch mit ihrer Schule und ihrer ersten Liebe schöne Erinnerungen verbinden, besser mit den ihm eigenen, optischen Signalen einholen. Das Thema der Möglichkeit von Pop jenseits der Mauer wird im Film am Ende eingefangen, weil nach dem kollektiven Befreiungstanz, der vor dem Schlagbaum Halt macht, die Farbe ausgeht und zu den Klängen «Dies Land ist dein Land» alles aus der erträumten Westernszene mit Indianern und Tumbleweed (im Wind transitorischer, verlorener Zeit) zurücksinkt ins triste Grau der Realität hinter der Mauer. Diese wehe Stimmung der bei aller Komödie und allem genregerechten Zusammenhalt in der Familie, wie bei einer Fernsehabendserie - Typen mit harmlosen Macken als sympathische Helden des Alltags - dennoch unüberhörbaren Gesell-schaftskritik im Untertext zeigt sich am deutlichsten, als Frau Kuppisch den Grenzübertritt mit falschem Pass nicht wagt: «Sie sah ein Pärchen, das zurück nach Westberlin wollte, und als Frau Kuppisch sah, wie locker und selbstbewusst die auftreten, wie laut die reden, wie gespielt die lachen und wie raumgreifend sie agieren [...], wusste sie, dass ihr zu einem Westler mehr fehlt als nur der Paß» (AS, S. 99). 


\section{NOTES}

1. Filmographie: Regie und Drehbuch: Tom Tykwer, Deutschland 1998, 81 Min. Produktion: Stefan Arndt, Bavaria Film International, Sony Picture Classics, X-Filme Creative Pool Production. Darsteller: Franka Potente, Moritz Bleibtreu, Nina Petri, Joachim Krol, Heino Ferch, Armin Rohde. Kamera: Frank Griebe, Schnitt: Mathilde Bonnefoy. Sound: Frank Behnke. Musik: Tom Tykwer, Johnny Klimek, Reinhold Hell. Deutscher Kritikerpreis, Ernst-Lubitsch-Preis, Bayer. Filmpreis, Großer Preis der Stadt Genf, Nominierung: Europ. Filmpreis, Goldener Löwe 1998/99.

2. Christine Ivanović: „Wende im Film? Vorläufiger Rückblick auf ein Jahrzehnt deutscher Einheit im Film“. In: Volker Wehdeking (Hg.): Mentalitätswandel in der deutschen Literatur zur Einheit (1990-2000). Berlin 2000, S. 225-235, hier S. 234.

3. Sabine Hake: «Film im wiederereinigten Deutschland. 1989 bis 2004». In: Dies: Film in Deutschland. Geschichte und Geschichten seit 1895. Reinbek 2004, Kap. 7, S. 303-340, hier S. 336.

4. Ulrike Draesner: Mitgift. Roman. München [2002], btb 2005, S. 332 f.- Cornelia Schleime: Weit fort. Roman, Hamburg: Hoffmann und Campe, 2008, S. 19.

5. Bayerischer Filmpreis, Deutscher Filmpreis 2006, Europäischer Filmpreis, Krakau 2006.

6. Filmographie: Regie: Florian Henckel von Donnersmarck. Deutschland 2006. 132 Min. Ko-Prod. des Bayerischen Rundfunks, Arte und Creado Film. DVD Buena Vista Home Ent. 2007. Darsteller: Martina Gedeck, Ulrich Mühe, Sebastian Koch, Ulrich Tukur. Musik (Sonate): Gabriel Yared.

7. Florian Henckel von Donnersmarck: Das Leben der anderen. Filmbuch. Frankfurt 2006, S. 169 f. Im folgenden Text zitiert als Sigle LA mit Seitenzahlen.

8. Vgl. das „Vorwort“ zu: Sonnenallee. Das Buch zum Farbfilm. Hg. von Leander Haußmann. Berlin: Quadriga 1999, S. 7.

9. Vgl. Michael Braun, Werner Kamp: Kontext Film. Beiträge zu Film und Literatur. Berlin 2006, «Vorwort», S. 7-10.

10. Zur positiven Rezeption, besonders des Films, vgl. Pflaum, H. G.: «Sonnenallee. Leander Haußmann, BRD». In: epd-Film, 16 (1999), H. 10, S. 34-35.- Moritz Baßler: «Thomas Brussig oder Die Erinnerung». In: Ders.: Der deutsche Pop-Roman. Die neuen Archivisten. München 2002, S. 46-68, hier bes. S. 67: «Erwartbarerweise ließ sich dieser Text sehr viel adäquater verfilmen als Helden wie wir [...], zumal er ansprechend besetzt ist: Rostocker Schauspielschüler geben die Jugendlichen, die weichgezeichnete Miriam [Teresa Weißbach, d.V.] ist so schön, dass Micha dagegen eine sehr unhollywoodeske [...] Körperlichkeit auf die Leinwand bringen darf. [...] All das funktioniert so lange reibungslos, wie der Film Situationskomödie sein darf. [...] Brussigs Buch gibt [...] eher die kleine Form vor».

11. Ebd., S. 24.

12. Thomas Brussig: Am kürzeren Ende der Sonnenallee. Berlin: Volk \& Welt, 1999, 157 S. Im folgenden Text mit Sigle AS und Seitenzahlen zitiert.

\section{RÉSUMÉS}

Die mit Tom Tykwers Film «Lola rennt» von 1998 beginnende Aufmerksamkeit für einen Jungen deutschen Film wird mit den Film-Entwicklungen seit dem Fall der Mauer zusammen gesehen und eine Auswahl aus den ca. dutzend neuen, wichtigen Filmen dieser Periode bis heute 
vorgeschlagen. Eingebettet wird diese Auswahl in Aspekte der Literaturadaption und der fiktionalen Gegenwartstendenzen. Die Auswahl zweier durch ihre Post-DDR-Perspektive östlicher und westlicher Tradition mitbegründete Qualität, Brussig / Haußmanns «Sonnenallee» (1999) und Von Donnersmarcks «Das Leben der anderen» (2006) erlaubt die Skizzierung einer Entwicklung und der zeitkritischen bis ,ostalgischen' Varianten des historischen Themas. Eine Rückkehr zu zeitkritischen Themen, diesmal eher einer Milleniumsperiode mit neuer Aufmerksamkeit für die Thematik der Terroristen (68er Gewalt und des 9. Sept. 2001) wird vorgeschlagen.

Le film de Tom Tykwer "Lola rennt", de 1998, qui attire l'attention sur le jeune cinéma allemand, est envisagé avec les évolutions du cinéma depuis la chute du Mur. On présente une sélection d'une douzaine de nouveaux films marquants de cette période jusqu'à aujourd'hui, en s'intéressant plus particulièrement aux aspects de l'adaptation d'œuvres littéraire et des tendances actuelles de la fiction. On s'est arrêté à deux films, "Sonnenallee ", de Brussig / Haußmann, (1999), et « Das Leben der anderen », de von Donnersmarck (2006), dont la qualité tient, entre autres, à la perspective de l'après-RDA restituée du point de vue de l'Est et de l'Ouest. L'analyse permet d'esquisser l'évolution et les variantes d'un même thème historique, dans une gamme de tons qui va de la critique politique jusqu'à l'ostalgie. On propose pour finir de retourner à des thèmes sensibles, cette fois plutôt à l'horizon du millénaire, avec un renouveau d'intérêt pour la thématique du terrorisme (violence de 1968 et du 11 septembre 2001).

\section{INDEX}

Mots-clés : cinéma

oeuvrecitee Das Leben der Anderen, Sonnenallee

\section{AUTEUR \\ VOLKER WEHDEKING}

Stuttgart Media University, HdM 